\title{
Neuroinflammatory gene expression analysis reveals potential novel mediators and treatment targets in interstitial cystitis with Hunner lesions
}

\author{
Glenn T. Werneburg ${ }^{1}$, Karen S. Keslar ${ }^{2}$, Paige Gotwald ${ }^{1}$, Johnathan Doolittle ${ }^{1}$, Sarah C. Vij ${ }^{1}$, \\ Byron H. Lee ${ }^{1}$, Daniel A. Shoskes ${ }^{1}$ \\ ${ }^{1}$ Department of Urology, Glickman Urological and Kidney Institute, Cleveland Clinic Foundation, Cleveland, OH, USA; ${ }^{2}$ Department of \\ Immunology, Lerner Research Institute, Cleveland Clinic Foundation, Cleveland, OH, USA \\ Contributions: (I) Conception and design: DA Shoskes; (II) Administrative support: P Gotwald; (III) Provision of study materials or patients: DA \\ Shoskes, J Doolittle, BH Lee, KS Keslar, SC Vij; (IV) Collection and assembly of data: DA Shoskes, GT Werneburg, KS Keslar; (V) Data analysis \\ and interpretation: DA Shoskes, GT Werneburg; (VI) Manuscript writing: All authors; (VII) Final approval of manuscript: All authors. \\ Correspondence to: Glenn T. Werneburg, MD, PhD. Department of Urology, Glickman Urological and Kidney Institute, Cleveland Clinic Foundation, \\ 9500 Euclid Ave, Q10-1, Cleveland, OH 44195, USA. Email: wernebg@ccf.org.
}

Background: We sought to study differential neuroinflammatory gene expression in men with interstitial cystitis (IC) with Hunner lesions compared with asymptomatic controls using NanoString, which uses barcoded probes to measure hundreds of genes. IC is a heterogenous condition lacking reliable biomarkers, and a subset of patients exhibits Hunner lesions, implicating the bladder as an inflammatory pain generator.

Methods: Blood, urine, and bladder biopsies were collected from 6 men with IC and Hunner lesions. 7 asymptomatic controls had blood and urine collected and 2 benign bladder biopsies were obtained from our tissue bank. RNA was isolated and analyzed with NanoString Human Neuroinflammation panel. Gene expression was considered significant if there was a $>1.5$-fold change and adjusted $\mathrm{P}$ value $<0.05$ compared with controls.

Results: Mean patient age was 61.5 years with 8 years median symptom duration. In bladder tissue, while many cytokine and chemokine genes had higher expression as expected (e.g., TNF , CXCL10), other significant genes included TRPA1 (1098-fold increased, expressed in pain sensing neurons) and TNFRSF17 (735-fold, B-cell related). In urine, there was 114-fold increase in S1PR4, which mediates pain via TRPdependent pathways. A patient on cyclosporine had lower inflammatory gene expression levels relative to other IC patients, but no difference in TRPA1.

Conclusions: Men with IC and Hunner lesions have a diverse set of neuroinflammatory genes with differential expression compared to controls. We identified genes linked to neuropathic pain through the TRP pathway and this expression was not reduced by cyclosporine. These findings open a new direction for biomarker and therapeutic discovery.

Keywords: Interstitial cystitis (IC); lower urinary tract symptoms (LUTS); bladder pain syndrome; biomarkers; Hunner lesions

Submitted Jul 27, 2021. Accepted for publication Sep 09, 2021.

doi: 10.21037/tau-21-657

View this article at: https://dx.doi.org/10.21037/tau-21-657

^ ORCID: 0000-0002-9518-672X. 


\section{Introduction}

Interstitial cystitis (IC) is characterized by pelvic pain, pressure, or discomfort related to the urinary bladder, often accompanied by urinary urgency or frequency, and relieved by voiding (1). IC is often debilitating, its etiology remains enigmatic, and it affects $2.7-6.5 \%$ of the U.S. population $(2,3)$. Patients with IC incur about $\$ 7,100$ in annual healthcare costs, nearly double the annual healthcare costs than patients without the condition (4). IC is a heterogenous condition, with no one-size-fits-all treatment, but rather treatment tailored to the individual. Commonly, patients are treated with a multimodal regimen of progressively escalated therapies, before they reach acceptable symptomatic control (5). Men are subject to misdiagnoses including prostatitis, benign prostatic enlargement, and epididymitis, and thus provide unique challenges practitioners (6). Due to this, along with higher prevalence of IC in women, few studies have focused on the condition in men. As yet there are no reliable and validated biomarkers to guide both diagnosis and treatment in this population.

There has been recent investigation of IC biomarkers with antiproliferative factor, epidermal growth factor, heparinbinding epidermal growth factor, glycosaminoglycans, and bladder nitric oxide as some of recent focus (7). However, due to difficulty or lack of practicality of assays, such as the need to culture bladder urothelial cells for detection of antiproliferative factor activity, diagnosis remains based on symptomatology and cystoscopic findings. An important subtype of IC is those patients with Hunner lesions found on cystoscopy. These patients are often older, have more severe symptoms and are more likely to respond to immunotherapy such as cyclosporine $(2,8)$. Nevertheless, diagnosis of Hunner lesions is inconsistent and prevalence can vary widely depending on the Urologist's experience identifying them. Therefore, identification of novel objective biomarkers would be helpful to guide effective therapy.

The NanoString nCounter is a novel rapid and automated platform allowing for direct mRNA measurement for up to 800 genes, using a small sample without the need for cDNA or polymerase chain reaction (9). We sought to compare RNA profiles of patients with IC with Hunner lesions relative to healthy controls. We utilized a predesigned cassette with nearly 800 genes associated with inflammation and/or neuropathic pain, and set out to study gene expression in blood, urine, and bladder tissue for this set of neuroinflammatory genes in men with IC compared to asymptomatic controls. Our approach allows for simultaneous measurement of gene interaction networks, as the orchestrated expression of interrelated genes, rather than any single gene, may play a key role in IC pathophysiology. We hypothesized that men with IC would have unique gene expression signatures associated with neuroinflammatory pathways, relative to asymptomatic controls. We present the following article in accordance with the MDAR reporting checklist (available at https:// dx.doi.org/10.21037/tau-21-657).

\section{Methods}

The study was approved by the institutional IRB (\#191515) and appropriate written consent was obtained for all subjects. The study was conducted in accordance with the Declaration of Helsinki (as revised in 2013). Blood, urine, and bladder biopsies were collected from 6 men with the clinical diagnosis of symptomatic IC found to have Hunner lesions (10). IC patients were excluded if they were not currently symptomatic with pain and lower urinary tract symptoms (LUTS), or if they had active infection (newly found or being treated with an antibiotic). IC patients were also excluded from our investigation if they were receiving immune-modulating treatments (e.g., corticosteroids), with the exception of cyclosporine for the secondary analysis of comparison of IC patients not on cyclosporine to the patient on cyclosporine. A single bladder biopsy was taken by an attending urologist with recognized expertise in the diagnosis and management of IC, from a visible Hunner lesion. The biopsy was obtained during cystoscopy under general anesthesia using cold cup biopsy forceps prior to lesion fulguration. At least $20 \mathrm{~mL}$ urine and approximately $3 \mathrm{~mL}$ blood were obtained either during a clinic visit or on the day of surgery prior to starting antibiotics or anesthesia. Urine and blood were similarly collected from 7 asymptomatic men, serving as controls, without signs or symptoms of UTI or LUTSs, presenting for a vasectomy. Benign biopsies were analyzed from our tissue bank, from 2 male patients with no documented history of LUTS or IC. These men underwent biopsy for suspicion of bladder cancer, and biopsies were taken from areas remote to any target lesion. Men were excluded from this group for evidence of UTI on preoperative urine culture, or other diagnosis of LUTSs or pain.

All samples were processed and frozen within 6 hours 
of collection. Samples were stored at the appropriate temperature $\left(-20{ }^{\circ} \mathrm{C}\right.$ for tempus tubes, $-80{ }^{\circ} \mathrm{C}$ for urine pellets, wherein RNA is stable indefinitely) for up to 9 months before being used for analysis. Three $\mathrm{mL}$ of blood was collected in Tempus Blood RNA tubes (Applied Biosystems; ThermoFisher Scientific), which contain a stabilizing reagent that immediately lyses blood cells and inactivates RNases. RNA isolation and analysis were performed as previously described (11). The RNA is stable indefinitely when stored at $-20{ }^{\circ} \mathrm{C}$. RNA was isolated from the blood samples using the Tempus Spin RNA Isolation Kit.

Urine samples were centrifuged at 3,000 $\mathrm{g}$ for 30 minutes at $4{ }^{\circ} \mathrm{C}$ in $50 \mathrm{~mL}$ conical tubes. The pellets were resuspended in $1 \mathrm{~mL}$ PBS, transferred to $2 \mathrm{~mL}$ microcentrifuge tubes and centrifuged at $10,000 \mathrm{rpm}$ for 4 minutes at room temperature. The PBS was removed and the urine pellets were then immediately stored at $-80^{\circ} \mathrm{C}$. RNA was isolated from the urine pellets using the Qiagen Rneasy Mini Kit. RNA from blood samples was quantified using a Nanodrop ND100 spectrophotometer. The mean RNA yield was $8.53 \mu \mathrm{g}$ (range, 3.5-13.6 $\mu \mathrm{g}$ ) and the OD260/280 ratios were all $>2.1$. RNA from urine samples was measured both with a Nanodrop ND100 spectrophotometer to determine the OD260/280 ratios, and the Qubit4 for RNA concentration. The mean RNA yield was $291 \mathrm{ng}$ (range, 0.1 to $1,600 \mathrm{ng}$ ) and the mean OD260/280 ratio was 1.8. A total of $100 \mathrm{ng}$ RNA was used for gene expression analysis with the 770gene NanoString Human Neuroinflammation gene panel.

\section{Statistical analysis}

Data were imported into ROSALIND ${ }^{\circledR}$ (https://rosalind. onramp.bio/) for quality control, normalization, analysis of fold-changes and $\mathrm{P}$ values, and identification of enriched pathways and gene sets. Differential gene expression was considered biologically and statistically significant if there was a greater than 1.5 -fold change compared to controls (the asymptomatic control group for the blood and urine samples, and the tissue bank group for the tissue sample), and Benjamini-Hochberg adjusted $\mathrm{P}$ value was $\mathrm{P}<0.05$. Patients were clinically phenotyped with the Urinary, Psychosocial, Organ-Specific, Infection, Neurologic/ Systemic and Tenderness (UPOINT) system (12). The authors are accountable for all aspects of the work in ensuring that questions related to the accuracy or integrity of any part of the work are appropriately investigated and resolved.

\section{Results}

In the IC group, mean patient age was 61.5 years (range, 44-69 years) and median symptom duration was 8 years (range, 2-17 years). Number of positive UPOINT domains ranged from 2-4 with a median of 2.5. In the control group, the mean patient age was 42.6 years (range, 30 55 years) and the patients had no reported history of LUTSs. The bladder biopsy patients were 48 and 65 years old, respectively.

\section{Bladder}

Bladder biopsy of the subjects with IC as compared to controls showed differential expression in 141 genes. The highest Nanostring gene set global significance scores were for apoptosis, adaptive immune response, and oligodendrocyte function (Table 1). The genes with the greatest significant positive fold-change were transient receptor potential cation channel, subfamily A, member 1 (TRPA1), tumor necrosis factor receptor superfamily, member 17 (TNFRSF17), lymphocyte antigen 9 (LY9) interferon regulatory factor 4 (IRF4), and chemokine (C-X-C motif) ligand 10 (CXCL10) (Table 2). The genes with the greatest significant negative fold-change were RNA binding protein, fox-1 homolog (C. elegans) 3 (RBFOX3), microtubule-associated protein tau (MAPT) (Table 2).

\section{Urine}

Urine of the subjects with IC as compared to controls showed differential expression in 123 genes. The highest gene set analysis scores were for Wnt (neuropathic pain), adaptive immune response, and angiogenesis (Table 1). The genes with the greatest significant positive fold-change were sphingosine-1-phosphate receptor 4 (S1PR4), suppressor of cytokine signaling 3 (SOCS3), colony stimulating factor 2 receptor, beta, low-affinity (granulocyte-macrophage) (CSF2RB), neutrophil cytosolic factor 1 (NCF1), and rasrelated $\mathrm{C} 3$ botulinum toxin substrate 2 (rho family, small GTP binding protein Rac2) (RAC2) (Table 3).

\section{Blood}

Blood of the subjects with IC as compared to controls showed differential expression in 41 genes. The highest gene set analysis scores were for oligodendrocyte function, carbohydrate metabolism, and matrix remodeling (Table 1). 
Table 1 Gene set analysis significance scores in bladder biopsy tissue, urine, and blood of IC patients versus healthy controls via NanoString Annotations

\begin{tabular}{|c|c|}
\hline Term & Significance score \\
\hline \multicolumn{2}{|l|}{ Bladder tissue } \\
\hline Apoptosis & 4.9464 \\
\hline Adaptive immune response & 4.9051 \\
\hline Oligodendrocyte function & 4.8428 \\
\hline Innate immune response & 4.6728 \\
\hline Cytokine signaling & 4.6176 \\
\hline NF-kB & 4.5874 \\
\hline Inflammatory signaling & 4.5428 \\
\hline Lipid metabolism & 4.3747 \\
\hline Wnt (neuropathic pain) & 4.3695 \\
\hline Insulin signaling & 4.1935 \\
\hline \multicolumn{2}{|l|}{ Urine } \\
\hline Wnt (neuropathic pain) & 3.0979 \\
\hline Adaptive immune response & 2.9616 \\
\hline Angiogenesis & 2.95 \\
\hline Insulin signaling & 2.9336 \\
\hline Inflammatory signaling & 2.8375 \\
\hline Carbohydrate metabolism & 2.8349 \\
\hline Oligodendrocyte function & 2.7838 \\
\hline Autophagy & 2.7356 \\
\hline Growth factor signaling & 2.6501 \\
\hline Innate immune response & 2.6483 \\
\hline \multicolumn{2}{|l|}{ Blood } \\
\hline Oligodendrocyte function & 1.8446 \\
\hline Carbohydrate metabolism & 1.6355 \\
\hline Matrix remodeling & 1.605 \\
\hline Neurons and Neurotransmission & 1.5946 \\
\hline Epigenetic regulation & 1.4982 \\
\hline Autophagy & 1.4736 \\
\hline Cellular stress & 1.4732 \\
\hline Astrocyte function & 1.471 \\
\hline Inflammatory signaling & 1.4521 \\
\hline Innate immune response & 1.448 \\
\hline
\end{tabular}

The genes with the greatest significant positive foldchange were lipocalin 2 (LCN2), CD68 molecule (CD68), granulin $(G R N)$, interleukin 1 receptor, type II (IL1R2), and pleckstrin homology domain containing, family $M$ (with RUN domain) member 1 (PLEKHM1) (Table 4). The genes with the greatest significant negative fold-change were radical S-adenosyl methionine domain containing 2 (RSAD2) and membrane-spanning 4-domains, subfamily A, member 1 (MS4A1) (Table 4).

\section{Cyclosporine: bladder, urine, and blood}

One of the IC patients was on cyclosporine, and thus we performed separate analyses comparing with the other IC patients. Bladder biopsy of the subject on cyclosporine as compared to those not on cyclosporine showed differential expression in 156 genes. Table 5 shows the genes with the largest difference between the patients not on cyclosporine versus the patient on cyclosporine in bladder, urine, and blood. As expected, key inflammatory genes including CD14 (501-fold in urine, 3-fold in blood), CXCL9 (248fold in urine) and CXCL10 (ten-fold in bladder tissue) were downregulated in the patient on cyclosporine. Notably, there was no significant difference in TRPA1 expression between the two groups.

\section{Discussion}

Progress in the treatment of IC has been slow, and hampered by a lack of predictive biomarkers and limited consensus on pathophysiology. Patients with Hunner lesions are a distinct subset of IC patients with documented inflammatory lesions in the bladder that may be responsive to fulguration, direct steroid injection and cyclosporine $(2,8)$. Our study used a novel technology to simultaneously measure the expression of neuroinflammatory genes in blood, urine and tissue between men with IC versus asymptomatic controls. While commonly known inflammatory markers were identified as expected, we found novel genes whose function could lead to new therapeutic interventions for IC. In bladder biopsies, we identified significantly different expression of 141 genes. As expected, many cytokine and chemokine genes had higher expression (e.g., CXCL10 92-fold higher, TNF 28-fold higher). Other significantly differently expressed genes included TRPA1 
Table 2 Genes with greatest significant differential expression (positive and negative change greater than ten-fold) in bladder tissue between IC patients and healthy controls

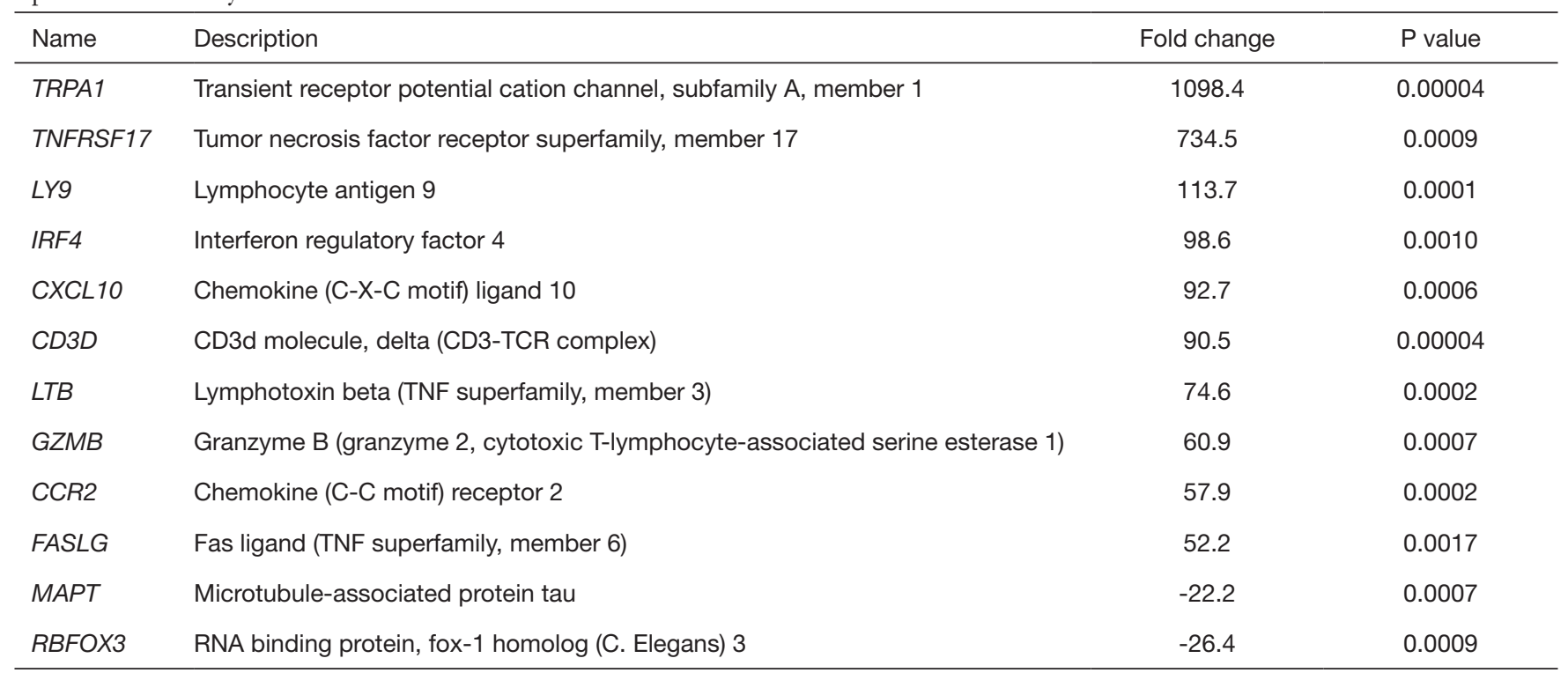

Table 3 Genes with greatest significant differential expression (positive and negative change greater than ten-fold) in urine between IC patients and healthy controls

\begin{tabular}{|c|c|c|c|}
\hline Name & Description & Fold change & $P$ value \\
\hline socs3 & Suppressor of cytokine signaling 3 & 45.5 & 0.0002 \\
\hline CSF2RB & Colony stimulating factor 2 receptor, beta, low-affinity (granulocyte-macrophage) & 37.0 & 0.0002 \\
\hline NCF1 & Neutrophil cytosolic factor 1 & 32.0 & 0.00008 \\
\hline WAS & Wiskott-Aldrich syndrome & 25.3 & 0.0002 \\
\hline SELL & Selectin L & 24.8 & 0.00009 \\
\hline LTB & Lymphotoxin beta (TNF superfamily, member 3 ) & 24.2 & 0.00007 \\
\hline ZBP1 & Z-DNA binding protein 1 & 23.8 & 0.0006 \\
\hline
\end{tabular}

(1,098-fold higher) and TNFRS17 (735-fold higher). Apoptosis, adaptive immune response, and oligodendrocyte pathways had higher significance scores in bladder tissue in patients with IC relative to controls. It is thought that neurogenic inflammation of the mucosa of the bladder can cause central and peripheral nervous system changes, which may be reflected in oligodendrocyte function, and can in turn contribute to IC (13).

TRPA1 was differentially expressed at 1000-fold greater levels in IC patients relative to asymptomatic controls. TRPA1 is an ion channel that has been shown to be an important component in pain pathways $(14,15)$. It has been implicated in inflammatory pain such as in osteoarthritis, neuropathic pain such as in diabetic neuropathy, migraine pain, and cancer pain. In addition, TRPA1 has also been shown to play an important role in "dysfunctional pain”, a category of pain with an ill-defined cause, where unexplained pain is the main presenting symptom (15). 
Table 4 Genes with greatest significant differential expression (positive and negative fold-change) in blood between IC patients and healthy controls

\begin{tabular}{llcr}
\hline Name & Description & Fold change & P value \\
\hline LCN2 & Lipocalin 2 & 9.4 & 0.0001 \\
CD68 & CD68 molecule & 2.9 & 0.0005 \\
GRN & Granulin & 2.4 & 0.0004 \\
IL1R2 & Interleukin 1 receptor, type II & 2.4 & 0.0204 \\
PLEKHM1 & Pleckstrin homology domain containing, family M (with RUN domain) member 1 & 2.3 & 0.0052 \\
CD14 & CD14 molecule & 2.3 & 0.0028 \\
FCER1G & Fc fragment of ige, high affinity I, receptor for; gamma polypeptide & 2.2 & 0.0011 \\
CD24 & CD24 molecule & 2.2 & 0.0231 \\
NRGN & Neurogranin (protein kinase C substrate, RC3) & 2.1 & 0.0147 \\
PTMS & Parathymosin & 0.0040 \\
MS4A1 & Membrane-spanning 4-domains, subfamily A, member 1 & 2.1 & -1.5 \\
RSAD2 & Radical S-adenosyl methionine domain containing 2 & -2.8 & 0.0396 \\
\hline
\end{tabular}

Dysfunctional pain conditions include IC, fibromyalgia, temporomandibular disorder, and irritable bowel syndrome.

TRPA 1 has been shown to be associated with inflammation and LUTS in the urinary bladder (16). A gain of function mutation in TRPA1 is responsible for familial episodic pain syndrome, and interestingly, antagonists have been shown to inhibit the gain-of-function mutant channel (17). Thus, TRPA1 may be an important drug target for pain syndromes. It has been shown that TRPA1 localized to unmyelinated nerve fibers in the urothelium, suburothelium, and muscle layers of the rat urinary bladder, and that activators of TRPA1 result in increased voiding frequency, decreased voided volume, and detrusor overactivity (18). It has also been shown to localize to the urothelial and interstitial cells of the urethra, and that TRPA1 agonists can modify the tone of human urethral preparations, suggesting a role in signaling in the human urinary outflow region (19). In a rat model of IC, inhibition of the TRPA1 decreased bladder hyperactivity and pain (20). It has also been shown to be differentially expressed in bladder tissue of those with IC (21). Our results build on these findings, and importantly, have demonstrated the presence of a significant upregulation in IC patients with Hunner lesions.

S1PR4, a G protein-coupled receptor never previously demonstrated in the urine, was detected at 114-fold higher expression in the urine of IC patients than healthy controls.
It is a member of the endothelial differentiation, G-proteincoupled (EDG) receptor family. It is mainly expressed in the hematopoietic system. A recent study utilizing S1PR4 knockout mice demonstrated that the main defect was in dendritic cell differentiation and cytokine secretion. In this study, dendritic cells were unable to switch $\mathrm{T}$ cells to Th17, and thus the immune response was shifted to a Th2 response (22). Of note, in IC, the Th17 cell-differentiation pathway has been shown to be significantly enriched in patients with IC with Hunner's lesions (23). Patients with IC are 100 times more likely to have inflammatory bowel disease than healthy individuals (24). Interestingly, severity of colitis was significantly reduced in a S1PR4 knockout inflammatory bowel disease mouse model (22). The molecule FTY720, which has a beneficial effect in treatment of colitis was postulated to act through the synergistic inactivation of both S1PR1 and S1PR4 (25). In a Phase II randomized controlled clinical trial, Etrasimod (APD334), an $\mathrm{S} 1 \mathrm{P} 1, \mathrm{~S} 1 \mathrm{P} 4$, and $\mathrm{S} 1 \mathrm{P} 5$ receptor modulator, was more effective than placebo in terms of clinical and endoscopic improvements in patients with moderate to severe ulcerative colitis (26).

Kittaka et al. have demonstrated a potential link between TRPA1 and S1PR (27). S1P-induced responses in the dorsal root ganglia neurons in mice were partially inhibited by TRPA1 antagonists. The group used a selective TRPA1 antagonist (HC-030031), and showed that it reduced the 
Table 5 Genes with greatest significant differential expression (positive fold-change) in bladder tissue, urine, and blood, between patients with IC not on cyclosporine versus the patient on cyclosporine

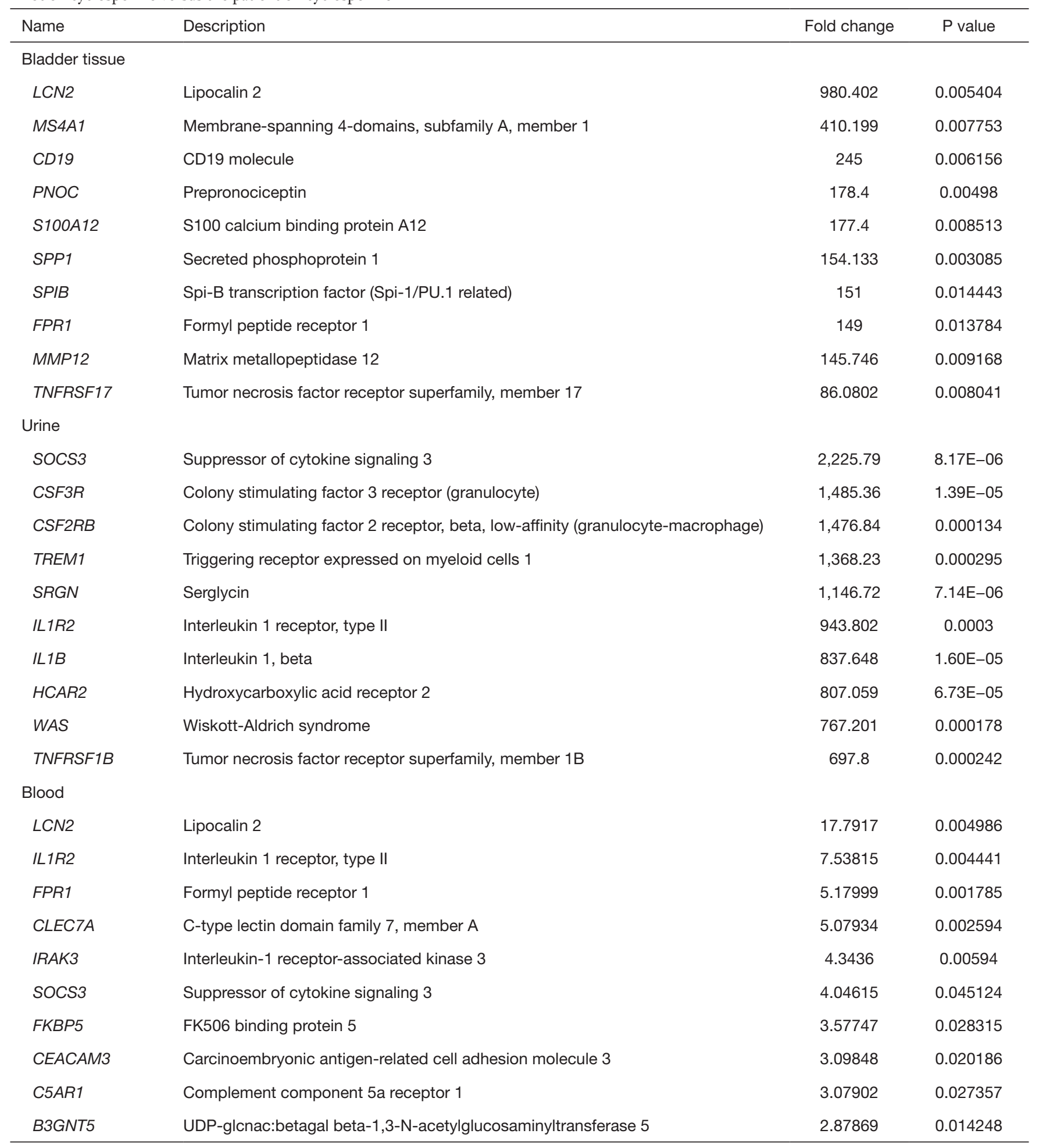


S1P-induced increases of intracellular calcium influx. Their results suggested that calcium influx caused by $\mathrm{S} 1 \mathrm{P}$ is mediated by TRPA1. Additional studies are needed to further understand these molecular underpinnings.

Our results, in the context of the above studies, elucidate a link between TRPA1 and S1PR in IC. TRPA1 has a 1,098-fold expression increase in bladder tissue and S1PR4 a 114-fold expression increase in urine in those with IC relative to asymptomatic controls. We propose that this combination may lead to an intracellular calcium influx and subsequent ATP release. Indeed, multiple studies have shown that ATP release in urothelial cells is controlled by a rise in intracellular calcium concentration $(28,29)$. It is known that ATP is released from the urothelium in the context of bladder stretch. In a feline model for IC, it has been shown that swelling-evoked ATP release in IC cells was elevated relative to cells of normal cats (30). Further, bladder urothelial cells in patients with IC had augmented extracellular ATP release relative to those of healthy controls (31).

TNFRS17, also known as B-cell maturation antigen $(B C M A)$, is preferentially expressed in mature $\mathrm{B}$ lymphocytes, and has been implicated in cell development and autoimmune response. Until now, it has not been detected in the bladder. TNFRS17 has been implicated in lymphomas, leukemias, and multiple myeloma, and autoimmune diseases including SLE. TNFRS17 has been shown to have inflammatory effects on myeloid cells, and to be expressed in macrophages (32). TNFRS17 has also been identified as a suitable candidate for CAR-T therapy in multiple myeloma (33).

One of our patients was on cyclosporine therapy. Although inflammatory gene expression levels were significantly lower, as expected, in blood, urine, and tissue, TRPA1 was not significantly decreased in the cyclosporine patient's bladder tissue. This finding, in the context of our other data and the literature, supports the importance of the identification of novel inhibitors of TRPA1 for the treatment of IC. Given that TRPA1 antagonists have been developed for a variety of pain conditions, there is the possibility that therapy delivered systemically or intravesically could improve symptoms in IC, sparing the long-term toxicities of cyclosporine.

Strengths of this study include the broad range of genes tested and inclusion of a healthy control group. Limitations include the relatively small sample size, a split between control samples for fluids and tissue, an age difference between groups, and lack of histological or proteomic analysis. The small sample size reflects the rarity of IC, particularly in the male population with Hunner lesions. Indeed, the female to male ratio is $5: 1$, the minority of these patients harbors Hunner lesions, and there has been minimal investigation in this population (34-36). Further, cyclosporine use in the population is exceedingly rare, as it is currently recommended as a fifth line treatment for IC by the American Urological Association (37). Our study population was confined to men, because of their unique challenges and misdiagnoses, the paucity of literature on men with IC, and because they are the focus of the clinical practice. The study calls for further investigations to generalize these findings to women and other patients with IC. Future work should also address the differential genetic signatures of men with IC as compared to those with chronic prostatitis, or benign prostatic hyperplasia, as there is overlap in these conditions and their differentiation in practice can be challenging. Further, the study was not designed to assess for outcomes of IC interventions based on genetic signatures, but this will be a future focus.

In conclusion, men with IC and Hunner lesions have a diverse set of neuroinflammatory genes with differential expression compared to controls. In addition to the expected inflammatory genes, we identified genes linked to neuropathic pain through the TRP pathway and this expression was not reduced by cyclosporine. These findings open a new direction for biomarker and therapeutic discovery.

\section{Acknowledgments}

Funding: This research was funded in part by generous philanthropic support at Cleveland Clinic. Special thanks to Mr. Sam Kellett and Mr. Paul Vitek for their support of our work. Nanostring analysis was performed by the NIAIDsponsored Clinical Trials in Organ Transplantation (CTOT) NanoString Core (U01 AI063594).

\section{Footnote}

Reporting Checklist: The authors have completed the MDAR reporting checklist. Available at https://dx.doi. org/10.21037/tau-21-657

Data Sharing Statement: Available at https://dx.doi. org/10.21037/tau-21-657

Conflicts of Interest: All authors have completed the ICMJE 
uniform disclosure form (available at https://dx.doi. org/10.21037/tau-21-657). DAS serves as an unpaid editorial board member of Translational Andrology and Urology from August 2020 to July 2022. DAS reports investment interest in Triurol. The other authors have no conflicts of interest to declare.

Ethical Statement: The authors are accountable for all aspects of the work in ensuring that questions related to the accuracy or integrity of any part of the work are appropriately investigated and resolved. The study was conducted in accordance with the Declaration of Helsinki (as revised in 2013). The study was approved by the institutional IRB (\#19-1515) and informed consent was taken from all the patients.

Open Access Statement: This is an Open Access article distributed in accordance with the Creative Commons Attribution-NonCommercial-NoDerivs 4.0 International License (CC BY-NC-ND 4.0), which permits the noncommercial replication and distribution of the article with the strict proviso that no changes or edits are made and the original work is properly cited (including links to both the formal publication through the relevant DOI and the license). See: https://creativecommons.org/licenses/by-nc-nd/4.0/.

\section{References}

1. Doggweiler R, Whitmore KE, Meijlink JM, et al. A standard for terminology in chronic pelvic pain syndromes: A report from the chronic pelvic pain working group of the international continence society. Neurourol Urodyn 2017;36:984-1008.

2. Crescenze IM, Tucky B, Li J, et al. Efficacy, Side Effects, and Monitoring of Oral Cyclosporine in Interstitial Cystitis-Bladder Pain Syndrome. Urology 2017;107:49-54.

3. Berry SH, Elliott MN, Suttorp M, et al. Prevalence of symptoms of bladder pain syndrome/interstitial cystitis among adult females in the United States. J Urol 2011;186:540-4.

4. Clemens JQ, Meenan RT, Rosetti MC, et al. Costs of interstitial cystitis in a managed care population. Urology 2008;71:776-80; discussion 780-1.

5. Colaco M, Evans R. Current guidelines in the management of interstitial cystitis. Transl Androl Urol 2015;4:677-83.

6. Arora HC, Shoskes DA. The enigma of men with interstitial cystitis/bladder pain syndrome. Transl Androl Urol 2015;4:668-76.
7. Kuo HC. Potential urine and serum biomarkers for patients with bladder pain syndrome/interstitial cystitis. Int J Urol 2014;21 Suppl 1:34-41.

8. Lai HH, Pickersgill NA, Vetter JM. Hunner Lesion Phenotype in Interstitial Cystitis/Bladder Pain Syndrome: A Systematic Review and Meta-Analysis. J Urol 2020;204:518-23.

9. Geiss GK, Bumgarner RE, Birditt B, et al. Direct multiplexed measurement of gene expression with colorcoded probe pairs. Nat Biotechnol 2008;26:317-25.

10. Gillenwater JY, Wein AJ. Summary of the National Institute of Arthritis, Diabetes, Digestive and Kidney Diseases Workshop on Interstitial Cystitis, National Institutes of Health, Bethesda, Maryland, August 28-29, 1987. J Urol 1988;140:203-6.

11. Shoskes DA, Keslar KS, Gotwald P, et al. Neuroinflammatory gene expression in chronic prostatitis/ chronic pelvic pain syndrome patients: insights into etiology and phenotype biology. Transl Androl Urol 2021;10:3340-7.

12. Nickel JC, Irvine-Bird K, Jianbo L, et al. Phenotypedirected management of interstitial cystitis/bladder pain syndrome. Urology 2014;84:175-9.

13. Kim DS. Potential Mechanisms Underlying the Increased Excitability of the Bladder Afferent Pathways in Interstitial Cystitis/Bladder Pain Syndrome. Urogenital Tract Infection 2018;13:26-34.

14. Bautista DM, Jordt SE, Nikai T, et al. TRPA1 mediates the inflammatory actions of environmental irritants and proalgesic agents. Cell 2006;124:1269-82.

15. Souza Monteiro de Araujo D, Nassini R, Geppetti P, et al. TRPA1 as a therapeutic target for nociceptive pain. Expert Opin Ther Targets 2020;24:997-1008.

16. Toktanis G, Kaya-Sezginer E, Yilmaz-Oral D, et al. Potential therapeutic value of transient receptor potential channels in male urogenital system. Pflugers Arch 2018;470:1583-96.

17. Kremeyer B, Lopera F, Cox JJ, et al. A gain-of-function mutation in TRPA1 causes familial episodic pain syndrome. Neuron 2010;66:671-80.

18. Streng T, Axelsson HE, Hedlund P, et al. Distribution and function of the hydrogen sulfide-sensitive TRPA1 ion channel in rat urinary bladder. Eur Urol 2008;53:391-9.

19. Gratzke C, Streng T, Waldkirch E, et al. Transient receptor potential A1 (TRPA1) activity in the human urethra--evidence for a functional role for TRPA1 in the outflow region. Eur Urol 2009;55:696-704.

20. Chen D, Liu N, Li M, et al. Blocking PAR2 Alleviates 
Bladder Pain and Hyperactivity via TRPA1 Signal. Transl Neurosci 2016;7:133-8.

21. Homma Y, Nomiya A, Tagaya $M$, et al. Increased mRNA expression of genes involved in pronociceptive inflammatory reactions in bladder tissue of interstitial cystitis. J Urol 2013;190:1925-31.

22. Schulze T, Golfier S, Tabeling C, et al. Sphingosine-1phospate receptor $4\left(\mathrm{~S} \mathrm{P}_{4}\right)$ deficiency profoundly affects dendritic cell function and TH17-cell differentiation in a murine model. FASEB J 2011;25:4024-36.

23. Akiyama Y, Maeda D, Katoh H, et al. Molecular Taxonomy of Interstitial Cystitis/Bladder Pain Syndrome Based on Whole Transcriptome Profiling by Next-Generation RNA Sequencing of Bladder Mucosal Biopsies. J Urol 2019;202:290-300.

24. Alagiri M, Chottiner S, Ratner V, et al. Interstitial cystitis: unexplained associations with other chronic disease and pain syndromes. Urology 1997;49:52-7.

25. Danese S, Furfaro F, Vetrano S. Targeting S1P in Inflammatory Bowel Disease: New Avenues for Modulating Intestinal Leukocyte Migration. J Crohns Colitis 2018;12:S678-86.

26. Sandborn WJ, Peyrin-Biroulet L, Zhang J, et al. Efficacy and Safety of Etrasimod in a Phase 2 Randomized Trial of Patients With Ulcerative Colitis. Gastroenterology 2020;158:550-61.

27. Kittaka H, DeBrecht J, Mishra SK. Differential contribution of sensory transient receptor potential channels in response to the bioactive lipid sphingosine-1phosphate. Mol Pain 2020;16:1744806920903515.

28. Matsumoto-Miyai K, Kagase A, Murakawa Y, et al. Extracellular $\mathrm{Ca} 2+$ regulates the stimulus-elicited ATP

Cite this article as: Werneburg GT, Keslar KS, Gotwald P, Doolittle J, Vij SC, Lee BH, Shoskes DA. Neuroinflammatory gene expression analysis reveals potential novel mediators and treatment targets in interstitial cystitis with Hunner lesions. Transl Androl Urol 2021;10(11):4100-4109. doi: 10.21037/ tau-21-657 release from urothelium. Auton Neurosci 2009;150:94-9.

29. Chess-Williams R, Sellers DJ, Brierley SM, et al. Purinergic receptor mediated calcium signalling in urothelial cells. Sci Rep 2019;9:16101.

30. Birder LA, Barrick SR, Roppolo JR, et al. Feline interstitial cystitis results in mechanical hypersensitivity and altered ATP release from bladder urothelium. Am J Physiol Renal Physiol 2003;285:F423-9.

31. Sun Y, Chai TC. Augmented extracellular ATP signaling in bladder urothelial cells from patients with interstitial cystitis. Am J Physiol Cell Physiol 2006;290:C27-34.

32. Kumar G, Maria Z, Kohli U, et al. CNS Autoimmune Responses in BCMA-Deficient Mice Provide Insight for the Failure of Atacicept in MS. Neurol Neuroimmunol Neuroinflamm 2021;8:e973.

33. Carpenter RO, Evbuomwan MO, Pittaluga S, et al. B-cell maturation antigen is a promising target for adoptive T-cell therapy of multiple myeloma. Clin Cancer Res 2013;19:2048-60.

34. Clemens JQ, Meenan RT, Rosetti MC, et al. Prevalence and incidence of interstitial cystitis in a managed care population. J Urol 2005;173:98-102; discussion 102.

35. Teichman JM. Hunner's lesions. Can Urol Assoc J 2009;3:478.

36. Gross J, Vetter J, Lai HH. Clinical Presentation of Urologic Chronic Pelvic Pain Syndrome (UCPPS) Varies With Presenting Age - Implication on Patient Evaluation. Urology. 2021 Jul 22:S0090-4295(21)00680-4.

37. Hanno PM, Erickson D, Moldwin R, et al. Diagnosis and treatment of interstitial cystitis/bladder pain syndrome: AUA guideline amendment. J Urol 2015;193:1545-53. 